\title{
Vers une valorisation des déchets ménagers en agriculture (péri) urbaine à Kolwezi : caractérisation et influence de la saisonnalité
}

\author{
Tshala Upite Joseph ${ }^{1}$, Kitabala Misonga Alain ${ }^{1 *}$, Tunda Mwamba Jean-Pierre ${ }^{2}$, Mufind Kayakez \\ Michel ${ }^{1}$, Kalenda Miji Arsene ${ }^{3}$, Kapele Kanyimbu Georges ${ }^{4}$, Nyembo Kimuni Luciens ${ }^{5}$. \\ ${ }^{1}$ Département de Phytotechnie, Faculté des Sciences Agronomiques, Université de Kolwezi, Kolwezi R.D. Congo. \\ 2Département de Zootechnie, Faculté des Sciences Agronomiques, Université de Kolwezi, Kolwezi R.D. Congo. \\ ${ }^{3}$ Ferme Agro-pastorale Tshabula, Kolwezi R.D. Congo \\ ${ }^{4}$ Département de Biochimie, Section Sciences Exactes, Institut Supérieur Pédagogique de Kolwezi, B.P 300, Kolwezi \\ R.D. Congo \\ ${ }^{5}$ Département de Phytotechnie, Faculté des Sciences Agronomiques, Université de Lubumbashi, B.P 1825; \\ Lubumbashi R.D. Congo \\ ${ }^{*}$ Correspondance, courriel : joellemisongaalain@gmail.com
}

Original submitted in on 24th January 2017. Published online at www.m.elewa.org on $30^{\text {th }}$ April 2017 https://dx.doi.org/10.4314/jab.v112i1.12

\section{RESUME}

Objectif : L'urbanisation et le développement économique ont généralement pour corollaire l'accroissement des besoins alimentaires et l'augmentation de la production des déchets dans les pays en voie de développement et dont la gestion demeure un problème. Cette étude s'est proposée d'apprécier d'une part les caractéristiques agronomiques d'un compost issu de déchets ménagers, de l'autre part, déterminer la part biodégradable pour une meilleure valorisation agricole à Kolwezi.

Méthodologie et résultats: Du 17 Mai 2015 au 17 Avril 2016, les déchets étaient collectés sur 100 ménages pris comme échantillon dans deux communes de la ville. Des poubelles étaient placées et à la fin de chaque semaine, ces poubelles étaient vidées, pesées et les tris des différentes catégories selon la nature étaient effectués. Le compostage était enfin réalisé pour la part biodégradable sur une durée de 4 mois à l'issue duquel les échantillons étaient pris pour les analyses chimiques. Les résultats ont révélé d'une part la présence de deux fractions de déchets à savoir: les biodégradables et les non biodégradables et d'autre part, la quantité des déchets variait selon les saisons et les communes. Pour une quantité moyenne mensuelle de $1001,5 \mathrm{~kg}$ par ménage, la fraction biodégradable représente $53 \%$ du poids. Par ailleurs, les concentrations en éléments majeurs étaient relativement élevées par rapport à celles observées au Cameroun pendant que celles en métaux lourds étaient largement inférieures par rapport aux normes internationales.

Conclusion et application des résultats : le compostage constitue une alternative de gestion de déchets, lesquels peuvent être valorisés en agriculture urbaine et périurbaine surtout dans un contexte où l'accès aux engrais synthétiques n'est pas à la portée de tous les maraichers de Kolwezi. Toutefois, pour une meilleure valorisation, une analyse microbienne serait nécessaire afin de prévenir des maladies.

Mots clés : Compost, Déchets ménagers, composition chimique, maraichère, Kolwezi. 
Enhancing the value of household waste for (peri) urban agriculture at Kolwezi: characterization and influence of seasonality

\section{ABSTRACT}

Objective: Urbanization and economic development are generally accompanied by the increase of waste production whose management and remains a problem in developing countries. This study to evaluate the characteristics of compost from household waste and to determine the biodegradable part for a better agricultural valorization in Kolwezi.

Methodology and results: From 17 May 2015 to 17 April 2016, waste was collected from 100 households sampled in two municipalities in the city. Dustbins were placed and at the end of each week, these trash could be emptied, weighed and the sorting of the different categories according to the nature were carried out. The composting was finally carried out for the biodegradable part over a period of 4 months at the end of which the samples were taken for the chemical analyzes. The results revealed, on the one hand, the presence of two fractions of waste, namely biodegradable and non-biodegradable and, on the other hand, the amount of waste varied according to the seasons and communes. For an average monthly quantity of $1001.5 \mathrm{~kg}$ per household, the biodegradable fraction represents $53 \%$ of the weight. Moreover, the concentrations of major elements were relatively high compared to those observed in Cameroon while those in heavy metals were much lower than the international standard.

Conclusion and application of results: composting constitutes an alternative waste management, which can be valued in urban and peri-urban agriculture especially in a context where access to synthetic fertilizers is not within the reach of all Kolwezi market gardeners. However, for better valorization, microbial analysis would be necessary to prevent disease.

Keywords: Compost, Household waste, chemical composition, vegetable garden, Kolwezi.

\section{INTRODUCTION}

L'urbanisation en Afrique est souvent associée d'un côté à un accroissement des besoins alimentaires dont la satisfaction devrait passer par l'intensification des activités agricoles et de l'autre côté, à l'augmentation de déchets dont la gestion n'a plus fait objet de planification (Chalot, 2004 ; Sotamenou, 2010). Pendant de nombreuses années, l'homme a cru qu'il suffisait de stocker le déchet à l'écart pour qu'il disparaisse définitivement, mais, c'était sans compter à l'environnement au sein duquel des phénomènes incessants de natures chimiques, biologiques finissent par entraîner et transformer le déchet ou certains de ses composants (Bruno, 1977). En effet, plusieurs auteurs (Ngnikam, 2003, Doublier, 2003 ; Rotich et al., 2006 ) soulignent que la gestion des déchets dans la plupart des pays en voie de développement plus précisément en Afrique est la plus mitigée et confrontée à des problèmes financiers, institutionnels (déficits d'organisation), techniques et physiques (absence de plan d'urbanisation), et par conséquent, ces pays sont confrontés à d'énormes problèmes sanitaires. En plus, la pollution par les déchets est de loin celle qui est la plus perçue par les citoyens, car contrairement à la pollution de l'air, du sol ou des ressources en eau, la pollution par les déchets est la plus « visible » à l'œil nu (Müller, 2007). La gestion de déchets constitue un défi majeur dès lors que leur production accroît ; en conséquence, de nouvelles filières d'élimination doivent être trouvées (Compaoré et al., 2010b). En outre, pour faire face à la demande alimentaire croissante, la plupart des agriculteurs des zones tropicales font recours aux fertilisants chimiques qui d'ailleurs restent très coûteux (Ayeni et al., 2010, Useni et al., 2013, Nyembo et al., 2014). Or, pour les sols sableux à sablo-argileux des zones semi arides tropicales d'Afrique Subsaharienne, il a été mis en évidence par plusieurs auteurs que les systèmes culturaux intensifs n'impliquant pas des niveaux élevés de restitutions organiques au sol, conduisaient à leurs dégradation et par conséquent une diminution de la productivité 

urbaine à Kolwezi : caractérisation et influence de la saisonnalité

végétale (Charreau et Nicou, 1971 ; Sedogo et al ., 1994; Omotayo et Chukwuka, 2009). Cette dégradation est souvent liée à une diminution des stocks organiques, alors que la gestion durable et la reconstitution des sols fortement dégradés constituent un défi pour l'agriculture des pays tropicaux (Mokossesse et al., 2009). Cette gestion signifie que les prélèvements doivent être compensés par des apports, de telle sorte que l'équilibre dynamique soit maintenu. Or pour l'Afrique, certains auteurs (Useni et al., 2013; 2014; Soudi, 2001) ont démontré que le compostage des déchets demeure une option écologiquement durable et économiquement attrayante. En plus, dans les villes africaines, les ordures ménagères sont composées en grande

\section{MATERIELS ET METHODES}

Milieu : Les déchets ont été récoltés dans les communes Dilala et Manika à Kolwezi, dans le sudouest en République Démocratique du Congo (26 $27^{\prime}$ E, 10¹1' S) (Famba et al., 2011). Depuis sa création, la ville de Kolwezi est habitée par une population qui affiche un taux de croissance continue près qu'environ $16 \%$ pour les nationaux. La ville compte près de 850000 habitants exerçant les activités industrielles et minières, mais aussi l'agriculture vivrière et maraichère (Dibwe, 2006). Le miombo, végétation dominante, mais en constante dégradation, pousse sur des sols acides relativement pauvres en matière organique (Kitabala et al., 2016). Le climat est du type Cw selon Köppen caractérisé par l'alternance de la saison de pluie et sèche (Malaise, 1973, Placet, 1962).

\section{Méthodologie}

Collecte des déchets : Les déchets ont été collectés du 17 Mai 2015 jusqu'au 17 Avril 2016 sur un échantillon de 100 ménages choisi systématiquement sur l'ensemble des communes. Ensuite, des sachetspoubelles ont été placés dans chaque ménage faisant partie de la zone de l'échantillon pour recueillir tous les déchets ménagers. A la fin de chaque semaine, ces poubelles étaient vidées et les tris des différentes catégories selon leur nature (Ghrabi et al., 2002)

\section{RESULTATS}

La quantité des déchets diffère d'une saison à l'autre c'est donc au cours de la saison de pluvieuse que les déchets biodégradables sont produits à une grande partie de matière organique (40 à $90 \%$ ) (N'Dayegamiye et al., 2005). En République Démocratique Congo, Mulaji, 2010 a montré l'importance d'utiliser un compost des déchets des ordures ménagères dans l'amélioration de la fertilisation des sols acides tropicaux de la région de Kinshasa. Au Burkina-Faso l'application du compost des ordures ménagères a avait aussi permis une nette amélioration des rendements des cultures maraîchères (Abdoulaye et Phal, 2007). Cependant, peu d'informations existent sur Kolwezi, et par conséquent la première étape de la valorisation de déchet reste la quantification et l'appréciation des valeurs fertilisantes de compost issu de déchets ménagers.

étaient effectués pour déterminer la composition physique des déchets d'une poubelle.

Caractérisation des déchets : Sur une durée de 4 mois, la part biodégradable a été compostée à la surface de sol (méthode en andain) (Mulaji, 2010). Les échantillons du compost séchés étaient broyés à l'aide d'un mortier en porcelaine puis tamisés sur un tamis de $2 \mathrm{~mm}$ pour les analyses chimiques et le dosage de la matière organique. Ainsi, le dosage de la matière organique était réalisé par la méthode de Walkley et Black (ISO, 2006). L'azote a été déterminé par la méthode de Kjeldahl modifié (Mulaji, 2010, ISO, 2006). Le dosage du phosphore total a été déterminé par méthode de Scheel. Elle se fait par spectrométrie en présence du molybdate de vanadium (ISO, 2006). Quant au phosphore disponible, il a été déterminé par la méthode de Bray, alors que l'extraction du potassium a été faite avec $0,1 \mathrm{~N}$ d'acide chlorhydrique $(\mathrm{HCl})$ et 0,4 $\mathrm{N}$ d'acide oxalique $\left(\mathrm{H}_{2} \mathrm{CnO}_{4}\right)$ et sa teneur a été déterminée au photomètre à flamme par la comparaison des intensités de radiations émises par les atomes de potassium (K) avec celles des solutions standards (Ouattara, 2014). Les éléments minéraux totaux ont été déterminés au spectrophotomètre par fluorescence de rayons $X$ après minéralisation totale de l'échantillon (Mulaji, 2010 ; Mpundu, 2010).

quantité soit une moyenne de $1001,5 \pm 88,9 \mathrm{Kg}$. Alors qu'au cours de la saison sèche la quantité de déchets biodégradables enregistrés a été de $383,93 \pm 57,7 \mathrm{Kg}$. II 
Tshala et al., J. Appl. Biosci. 2017 Vers une valorisation des déchets ménagers en agriculture (péri) urbaine à Kolwezi : caractérisation et influence de la saisonnalité

en de même pour les deux communes, ces moyennes enregistrées sont de 795,5 $\pm 395,4 \mathrm{Kg}$ enregistré dans la

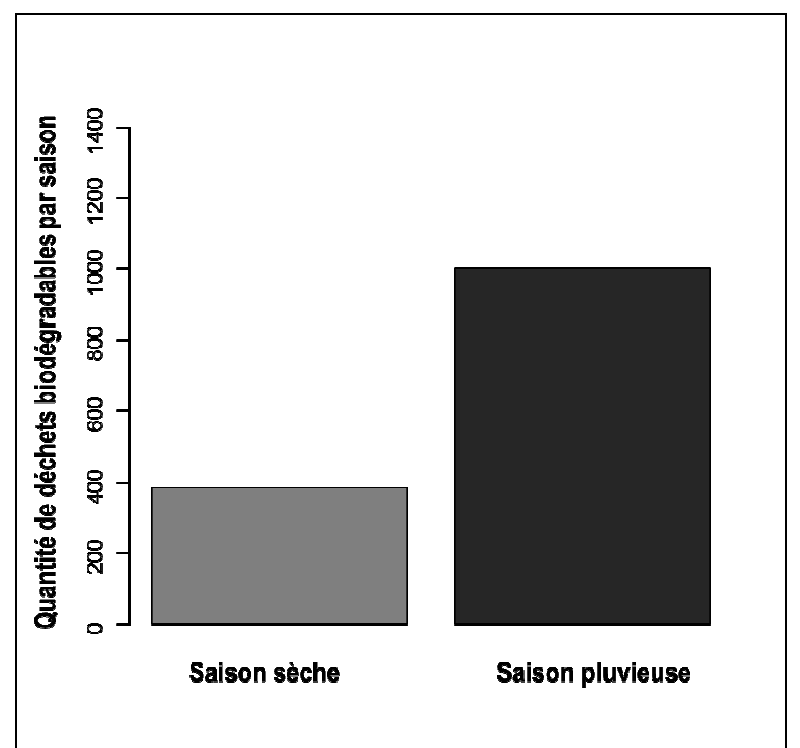

Figure 1 : La moyenne générale de déchet biodégradable selon les saisons et cités commune Dilala et $668,5 \pm 331,3 \mathrm{Kg}$ enregistré dans la Manika.

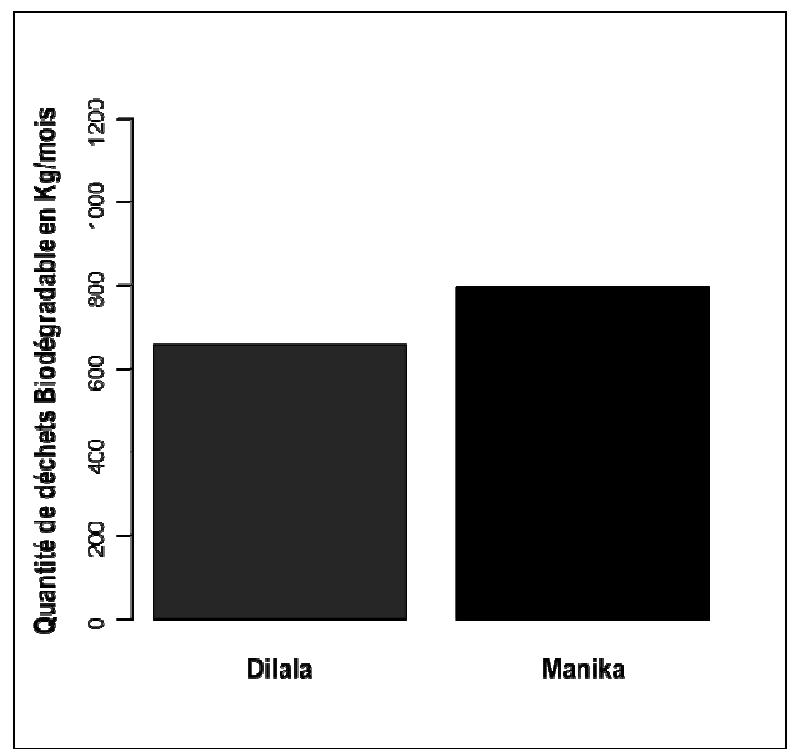

II ressort de cette étude que quel que soit la commune, la quantité des déchets non biodégradable ne diffère pas, par contre elle supérieure en saison de pluie qu'en saison sèche.
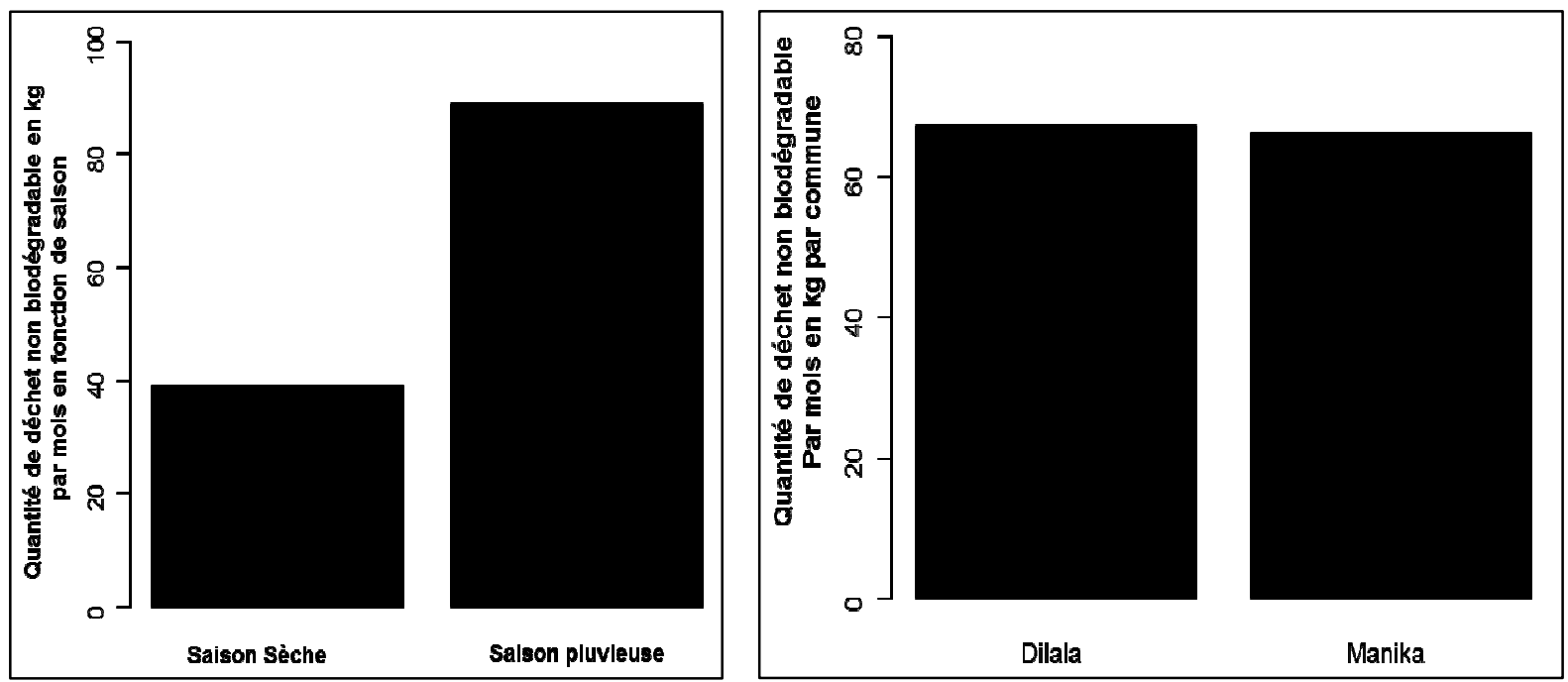

Figure 2 : La moyenne générale de déchet non biodégradable selon les saisons et cités 


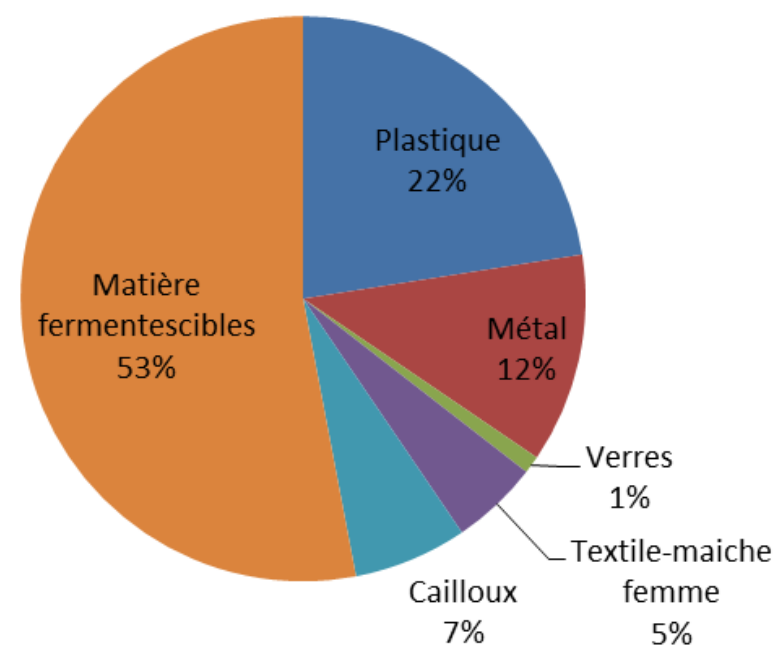

Figure 3. Composition moyenne d'une poubelle de déchets ménagers à Kolwezi

Au regard des pourcentages donnés dans le graphique, il ressort qu'en moyenne une poubelle d'un ménage à Kolwezi est constituée de $53 \%$ des matières biodégradables alors que la part non biodégradable ne constitue que $47 \%$ de la composition moyenne de la poubelle. Ce résultat constitue un paramètre important quant au choix du mode de traitement de ces déchets par exemple les possibilités de compostage jusqu'ici peu et/ou non exploitées dans la ville de Kolwezi.

Le Tableau 1 Teneurs en éléments traces métalliques ( $\mathrm{Fe}, \mathrm{Cu}, \mathrm{Mn}, \mathrm{Zn}, \mathrm{Pb})$ dans l'échantillon du compost ménager à Kolwezi.

\begin{tabular}{llllll}
\hline \multirow{2}{*}{ chantillon } & $\mathrm{Fe}$ & $\mathrm{Mn}$ & $\mathrm{Zn}$ & $\mathrm{Cu}$ & $\mathrm{Pb}$ \\
\cline { 2 - 6 } & $\%$ & $\mathrm{mg} \mathrm{kg}^{-1}$ & $\mathrm{mg} \mathrm{kg}^{-1}$ & $\mathrm{mg} \mathrm{kg}^{-1}$ & $\mathrm{mg} \mathrm{kg}^{-1}$ \\
\hline Compost & 1,63 & 0,298 & 0,229 & 0,267 & 0,19 \\
\hline Normes Standards $\mathbf{~ m g ~ k g}^{-1}$ & & & 300 & 100 & 100 \\
\hline
\end{tabular}

$\mathrm{Au}$ regard des chiffres donnés dans le tableau précédent, les concentrations des ETM sont relativement moins importantes et restent en dessous des valeurs limites des normes standards. Pour dire qu'elles sont non dangereuses pour les plantes et les humains.

Tableau 2. Comparaison des teneurs en éléments chimiques aux valeurs obtenues au Cameroun et quelques normes internationales.

\begin{tabular}{l|l|l|l|l|l|l|l|l|l}
\hline Source & $\mathbf{p H}$ & $\mathbf{C O}$ & $\mathbf{M O}$ & $\mathbf{N}$ & $\mathbf{C / N}$ & $\mathbf{P}$ disponible & $\mathbf{K}$ & $\mathbf{C a}$ & $\mathbf{C l}$ \\
\cline { 2 - 12 } & & $\%$ & $\%$ & $\%$ & & $\mathbf{m g ~ k g}^{-1}$ & $\%$ & $\%$ & $\%$ \\
\hline Cette étude & 7,4 & 7,15 & 12,33 & 0.57 & 13 & 395 & 0.69 & 1.70 & 0.17 \\
\hline Norme AFNOR & & & & $>0,25$ & $<20$ & - & $>1$ & & \\
\hline Norme FAO & & & & $0,4-0,5$ & $15-20$ & - & $0,4-2,3$ & & \\
\hline YE (2007) & 8,88 & 4,01 & 6,91 & 0,33 & 12,4 & 28,65 & 0,00169 & 0,0191 & - \\
\hline $\begin{array}{l}\text { Compaore et al. } \\
\text { 2010a. }\end{array}$ & 7,3 & 4,07 & 7,01 & 0,36 & 11,30 & 27,6 & 1,26 & - & - \\
\hline
\end{tabular}

Les résultats des analyses chimiques confirment que le compost de déchets présente des teneurs élevées en éléments de fertilité et sont conformes aux critères de la norme prescrite par la FAO et AFNOR. En effet, la concentration en carbone organique est de $7,15 \%$ ce qui conduit à une teneur en matière organique de 

urbaine à Kolwezi : caractérisation et influence de la saisonnalité

$12,33 \%$. Le compost contient une teneur de $0,57 \%$ en azote total, un rapport de $\mathrm{C} / \mathrm{N}$ de 13 et est cependant compris entre la gamme normale prescrite la FAO. Le phosphore disponible est de $395 \mathrm{mg} \mathrm{kg}^{-1}$ soit une

\section{DISCUSSION}

L'habitude et mode alimentaire de la population ainsi que le climat aurait une influence sur la quantité de déchets dans le deux communes à Kolwezi. II se révèle aussi que la quantité de déchets bio et/ou non biodégradable diffère d'une commune à l'autre probablement à cause du niveau de vie de la population. Des conclusions similaires ont été prouves à Yaoundé au Cameroun où la production moyenne des déchets ménagers est de $0,6 \mathrm{~kg} / \mathrm{hab}$. jour en saison sèche contre $0,98 \mathrm{~kg} / \mathrm{hab} / \mathrm{jour}$ en saison de pluie (Charnay, 2005 ; Aina, 2006). Les moyennes obtenues s'apparentes aux conclusions des plusieurs travaux réalisés en Afrique de l'Ouest notamment celui de Meunier-Nikiema (2007). L'unique hypothèse serait l'augmentation de la population observée ces dernières années suivant les conclusions d'Agdag (2008) ; Sharholy et al. (2007); Ahmed \& Ali (2006). Une étude menée par Ngoran (1993), a montré aussi que l'augmentation et l'intensification des activités socioéconomiques de la population auraient une incidence considérable sur la production des déchets dans les pays en voie de développement. Une bonne planification d'un système de gestion des déchets ménagers exige au préalable une bonne connaissance de la qualité et des quantités produites dans le présent et une estimation correcte des prévisions dans le futur (Ngoran, 1993). Les résultats obtenus sur la part fermentescibles justifient pleinement que la fabrication du compost et/ou valorisation agronomique du compost issu de déchets ménagers pour les maraichers de la

\section{CONCLUSION}

Les enquêtes constituent un bon moyen de collecte des informations sur la quantité et la qualité des déchets produits. Couplés aux analyses chimique et physique, les résultats obtenus révèlent en moyenne qu'une poubelle d'un ménage à Kolwezi est constituée d'une bonne part des déchets biodégradable soit $53 \%$, ce qui constitue un paramètre important quant au choix du mode de traitement des déchets par exemple le compostage jusqu'ici peu et/ou non exploitées dans la ville, bien plus l'accès aux engrais synthétique n'est pas à la portée de tous. Sa fabrication réduirait considérablement le volume des déchets mise en disponibilité de 7,58\%. II contient également $0,69 \%$ de potassium et $1,70 \%$ de calcium tandis que la concentration du chlore est de $0,17 \%$.

nouvelle province de Lualaba est possible aussi longtemps que l'accès aux engrais synthétique n'est pas à la portée de tous. Dans la plupart de cas, en moyenne, une poubelle est constituée souvent de 50 à $60 \%$ des déchets putrescibles, ce que confirment également Rese et al. (1997). Le taux de la part fermentescible est plus élevé que les autres composants. Cela s'explique par la nutritionnel des habitants qui favorisent la consommation des légumes en raison de leurs pouvoir d'achat. Les concentrations obtenues sur l'ensemble des éléments chimiques sont comprises entre la gamme établie par FAO et AFNOR. Selon les conclusions de Compaore et al., 2010a, les teneurs en éléments minéraux des composts sont beaucoup liées à la nature des déchets et en teneur initiale des éléments majeurs (Soudi, 2001). Les valeurs en $\mathrm{Fe}, \mathrm{Cu}, \mathrm{Mn}, \mathrm{Zn}, \mathrm{Pb}$ obtenues sont inférieures aux valeurs indicatives (seuils limites) de toxicité dans les sols agricoles telles que rapportées par la norme standard. Mais leurs présences seraient probablement justifié par les piles, batteries, peintures, médicaments...) tel que démontré par (Biney et al., 2006 cités par Yé, 2007). Néanmoins ces teneurs en métaux lourds dans cette étude sont largement inférieures à celles obtenues par (Guene, 1995 ; Wass 1996) dans les déchets solides de Ouagadougou. La gestion des déchets à Kolwezi serait toute opération relative à la collecte, au transport, à la valorisation et à l'élimination.

décharge et serait une voie nouvelle de gestion des déchets ménagers. L'analyse croisée des résultats obtenus sur concentration des éléments majeurs a attesté que le compost pourrait constituer une alternative moins couteuse que l'utilisation des engrais chimiques dans maraichage. Toutefois, pour une meilleure valorisation agricole, une analyse microbiologique s'impose en vue de réduire les risques d'exposition humaine et un essai évaluant sa qualité nutritionnelle sur la croissance et rendement des cultures sont nécessaires. 


\section{BIBLIOGRAPHIE}

Abdoulaye S. \& Phal M., 2007. Valorisation agricole des ordures ménagères en zone soudanosahélienne : cas de la ville de Bobo Dioulasso. In Sciences et Médecine, Revue. CAMESSérie (A) 05 : 64-70

Agdag N. O., 2008. Comparison of old and new municipal solid waste management systems in Denizli, Turkey. Waste Management, 2008

Ahmed A.S. \& Ali M., 2006. People as partners: Facilitating people's participation in publicprivate partnerships for solid waste management. Habitat International 30: 781-796

Aina, M.P., 2006. Expertises des centres d'enfouissement techniques des déchets urbains dans les PED: contribution à l'élaboration d'un guide méthodologique et à sa validation expérimentale sur site. Thèse. Université de Limoges.

Ayeni L .S \& Adetunji M.T., 2010. Integrated application of poultry manure and mineral fertilizer on soil chemical properties, nutrient uptake, yield and growth components of maize. Nature and Science 8(1): 60-37

Bruno D, 1977. Systèmes d'aide à la décision pour le traitement des déchets industriels spéciaux. Thèse de doctorat, STD, INSA de Lyon, 313p

Charnay, F., 2005. Compostage des déchets urbains dans les pays en développement: Elaboration d'une démarche méthodologique pour une production pérenne de compost. Thèse, Université de Limoges. $277 \mathrm{p}$

Charreau, C. et R. Nicou. 1971. L'amélioration du profil cultural dans les sols sableux et sablo argileux de la zone tropicale sèche ouest-africaine et ses incidences agronomiques. Bulletin Agronomique IRAT $\mathrm{N}^{\circ} 23$, Paris.

Chalot F., 2004. De l'amont vers l'aval : l'émergence d'une filière de gestion des déchets adaptée aux villes africaines. Synthèse et analyse des actions relatives aux déchets. In Gestion durable des déchets et de l'assainissement urbain. Mars 2004, pp 45-69.

Compaoré E. \& Nanéma L.S., 2010b. Compostage et qualité $\mathrm{d}$ compost des déchets urbains solides de la ville de Bobo Dioulasso, Burkina Faso. Tropicultura 28 (4) : 232-237

Compaoré E., Nanema L.S., Bonkoungou S., Sedogo M.P., 2010a. Évaluation de la qualité de composts de déchets urbains solides de la ville de Bobo-Dioulasso, Burkina Faso pour une utilisation efficiente en agriculture. Journal of Applied Biosciences 33 :2076-2083.

Dibwe dia Mwembu D., 2006. Le travail des enfants dans les mines et carrières au Katanga Lubumbashi: Rapport des recherches effectuées durant la dix-septième session des travaux de I'Observatoire du Changement Urbain (OCU), Coopération universitaire au développement (CUDBelgique).

Doublier G., 2003. Tri sélectif et valorisation des déchets urbains de la ville de N'djaména(Tchad). Rapport final, février 2003, $70 \mathrm{p}$.

Fwamba F., Jean-Pierre M., Henry M. \& Ernest M., 2011. De la Gécamines Providence à la Gécamines partiellement Privatisée "Gécamines ndjo Baba, Gécamines ndjo Mama». Rapport d'enquête, $87 \mathrm{p}$

Ghrabi A, R'houma A, Ennabli M \& Picoud F., 2002. Caractérisation des déchets ménagers dans la région du Sahel de la Tunisie. EPCOWM, 260270.

Guene O., 1995. Compostage artisanal intégré à la gestion des ordures ménagères, données de terrain et outils de diffusion. CREPA, Ouagadougou (Burkina Faso), 68p

ISO 10694, 2006. Part III a Sampling and Analysis of Soil - Determination of organic matter content. ISO, Geneva.

Kitabala M.A., Tshala U.J., Kalenda M.A., Tshijika I.M \& Mufind K.M., 2016. Effets de différentes doses de compost sur la production et la rentabilité de la tomate (Lycopersicon esculentum Mill) dans la ville de Kolwezi, Province du Lualaba (RD Congo). Journal of Applied Biosciences, 102: $9669-9679$

Malaisse F. 1973 : Caractérisations climatiques et écologiques du Katanga (République du Zaïre) bulletin agronomique de Gembloux, Hors-série 1972 : $140-150 \mathrm{pp}$

Meunier-Nikiema A., 2007. Géographie d'une ville à travers la gestion des déchets: Ouagadougou (Bourkina Faso), in revue "M@ppemonde ", № 87 (3-2007), Maison de la Géographie, Montpellier.

Mokossesse J.A., Lepage M. \& Josens G., 2009. Croissance en pots de quatre espèces végétales sur des substrats enrichis avec la terre de termitières de Cubitermes. Tropicultura, 27(3) :168-175

Mpundu M.M.M., 2010. Contaminations des sols en 
Eléments Traces Métalliques à Lubumbashi (Katanga/RDC). Évaluation des risques de contamination de la chaîne alimentaire et choix de solutions de remédiations. Thèse de doctorat, Faculté des sciences agronomiques, Université de Lubumbashi, Inédit, $401 p$

Mulaji K.C., 2010.Utilisation des composts de biodéchets ménagers pour l'amélioration de la fertilité des sols acides de la province de Kinshasa (République Démocratique $d u$ Congo). Thèse de doctorat, Université de Liège- Gembloux Agro-Biotech, 220p.

Müller, C., 2007. Anaerobic Digestion of Biodegradable, Solid Waste in Low- and Middle Income Countries Overview over existing technologies and relevant case studies. Dübendorf: EAWAG \& SANDEC, $63 p$.

N'Dayegamiye, A., Drapeau, A., Laverdière, M.R., 2005. Effets des apports de composts de résidus ménagers sur les rendements des cultures et certaines propriétés du sol. Agrosolutions 16 (2) :57-71.

Ngnikam E., 2003. Mise en place des structures de précollecte et de traitement des déchets solides urbains dans une capitale tropicale, Yaoundé (Cameroun). Rapport final, Janvier 2003, 186 p. + Annexes.

Ngoran K. C., 1993. Gestion des déchets ménagers dans la ville d'Abidjan. Bulletin du CIEH N ${ }^{\circ} 94$, 1993 octobre, $10 p$

Nyembo K.L., Useni S.Y., Chinawej M. M. D, Kyabuntu I.D, Kaboza Y., Mpundu M.M, Baboy L.L., 2014. Amélioration des propriétés physiques et chimiques du sol sous l'apport combiné des biodéchets et desengrais minéraux et influence sur le comportement du maïs (Zea mays L. variété Unilu). Journal of Applied Biosciences 74:6121-6130

Omotayo, O.E. \& Chukwuka, K.S., 2009. Soil fertility restoration techniques in sub-Saharan Africa using organic resources. African Journal of Agricultural Research 4: 144-150.

Ouattara S., 2014. Diagnostic des pratiques de valorisation agronomique de substrats organiques dans la zone urbaine et périurbaine de la ville de Bobo-Dioulasso (Burkina Faso). Mémoire de fin de cycle, $77 \mathrm{p}$.

Placet, 1962. Région de Kolwezi, monographie hydrogéologique, Département de géologie, Gécamines (éd) (République du Zaïre, 172p

Rese A., Giquel L. \& Rivière D., 1997. Compostage des déchets fermentescibles issus des collectes séparatives. Application de deux procédés. Ademe et Cemagref, 89p et annexes.

Rotich K. H., Yonsheng Z \& Jun D., 2006. Municipal solid waste management challenges in developing countries - Kenyan study in Waste management 26: 92-100.

Sedogo P.M., Lompo F. \& Ouattara B., 1994. Le carbone et l'azote dans les différents fractions granulométriques d'un sol ferrugineux tropical: Effet de quatre types d'amendement organique. Science et technique. Sciences naturelles $\mathbf{2 1}$ (01):114-124

Sotamenou J. 2010. Le compostage : une alternative soutenable de gestion publique des déchets solides au Cameroun. Thèse en Economie, Université de Yaoundé II, Faculté des Sciences Économiques et de Gestion, Cameroun, 364p

Sharholy M., Ahmad K., Vaishya R.C. \& Gupta R.D, 2007. Municipal solid waste characteristics and management in Allahabad, India. Waste Management 27: 490-496.

Soudi B., 2001. Compostage des déchets ménagers et valorisation du compost : cas des petites et moyennes communes au Maroc. éd Actes, $104 p$.

Useni S.Y., Chukiyabo K.M., Tshomba K.J., Muyambo M.E., Kapalanga K.P., Ntumba N.F., Kasangij K.P., Kyungu K.A., Baboy L.L., Nyembo K.L. \& Mpundu M.M., 2013. Utilisation des déchets humains recyclés pour l'augmentation de la production du maïs (Zea mays L.) sur un ferralsol du sud-est de la RD Congo. Journal of Applied Biosciences 66: 5070 - 50811

Useni S.Y., Kanyenga L. A., Assani B.L., Ekondo O.A., Baboy L.L., Ntumba K. B., Mpundu M. M. \& Nyembo K L., 2014. Influence de la date de semis et de la fertilisation inorganique sur le rendement de nouveaux hybrides de maïs (Zea mays L.) à Lubumbashi. Journal of Applied Biosciences 76:6316- 6325

Waas. E., 1996: Valorisation des déchets organiques dans les quartiers populaires des villes africaines. Projet FNRS N5001-038104. Module 7, développement et environnement. Programme prioritaire SKAT. 143p.

Yé L., 2007. Caractérisation des déchets urbains solides utilisables en agriculture urbaine et périurbaine : cas de Bobo-Dioulasso. Mémoire de DEA, Université Polytechnique de BoboDioulasso (Burkina Faso), 48p. 